Proc. Indian Acad. Sci. (Earth Planet. Sci.), Vol. 93, No. 2, July 1984, pp. 111-116.

(C) Printed in India

\title{
Wind dependence of quasi-specular sea scatter
}

\author{
ABHIJIT SARKAR and LAKSHMI BHADURI \\ Meteorology Division, Space Applications Centre, Ahmedabad 380053, India
}

\begin{abstract}
Earlier investigations have shown that a power law relationship of the type $\sigma=a W^{b}$ exists between the scattering coefficient $\sigma$ and sea surface wind speed $W$ at microwave frequencies. The coefficients $a$ and $b$ are usually quoted for different frequencies, look angles, polarizations etc. This paper attempts to define the angular dependence using such a power law relationship in the quasi-specular range.
\end{abstract}

Keywords. Radar scatterometer; scattering coefficient; sea surface wind speed.

\section{Introduction}

A potential application of the radar scatterometer as a remote sensor is in measuring the surface wind speed over the ocean. Some of the earliest experiments conducted to measure the radar return of the sea were airborne radar flights conducted by the Naval Research Laboratory of USA in 1965. Since then various experiments like NASA [ Johnson Space Centre flight experiments, NASA] Langley Research Centre flight experiments, Skylab S-193 satellite experiment and finally experiments with radar scatterometer on SEASAT (Moore and Fung 1979; Jones and Schroeder 1978) have confirmed the dependency of scattering coefficient on ocean surface wind speed. The observation parameters in these experiments varied. The frequencies ranged from 0.4 to $14 \mathrm{GHz}$, the angle of incidence from $0^{\circ}$ (vertical) to almost $90^{\circ}$ (near grazing) at all possible cases of polarization combinations.

Theories explaining the radar backscatter from sea have been encouraging and are moderately successful in explaining experimental results. This is amply demonstrated by the results of the SEASAT scatterometer (Jones et al 1982). Fung (1981), Barrick and Peake (1968) and others have examined and reviewed various theories for scattering of electromagnetic waves from rough sea surfaces. Radar backscattering from the sea for angles of incidence beyond about $20^{\circ}$ is governed by the Bragg scattering mechanism. The radar wavelengths normally used are resonant to components of the ocean surface that are either very short gravity waves or surface tension waves. In contrast, at nearvertical incidence angles, the signals returned by the surface are dominated by those produced by the geometric optics or physical optics mechanisms. In this region the slopes are such that one can achieve specular reflection from facets on the sea surface, having narrow backscattering patterns.

A generalised wind-backscatter relation which will take care of the variation in all observation parameters viz the angle of incidence, frequency, polarization etc, is yet to emerge. This paper explores the possibility of generalisation of the wind-backscatter relation with respect to the variation of incidence angle. The data base used for the present study was generated from the theoretical model of Barrick (1974), which is applicable to angles near the vertical (quasi-specular range of angles). 


\section{Scattering in the quasi-specular region and the specular point model}

The central theme in the application of the theory of scattering lies in the description of the interaction of electromagnetic waves in the microwave range with the surface of the sea. Various investigations (e.g. Kitaigorodskii 1981; Bass et al 1968) conducted in this field have shown that the interaction has a resonant nature, i.e. only certain fourier components of the surface shape are responsible for scattering in a given direction. This mechanism, known as Bragg scattering, is predominant for incidence angles above about $20^{\circ}$. However, at near-vertical incidence angles the primary mechanism is quasi specular in which geometric or physical optics techniques can be used to develop theoretical results.

A simplified form of the backscatter cross-section per unit area $\sigma^{\circ}$ in the quasispecular region (i.e. with angles of incidence up to $15-20^{\circ}$ ) is given by Barrick (1974):

$$
\sigma^{\circ}=\pi \sec ^{4} \theta p_{s}(-\tan \theta, 0)|R(0)|^{2}
$$

where $\theta$ is the angle of incidence from the vertical; $p_{s}\left(\zeta_{x}, \zeta_{y}\right)$ the joint probability density function of the surface slopes $\zeta_{x}\left(=\frac{\partial \zeta}{\partial x}\right)$ and $\zeta_{y}\left(=\frac{\partial \zeta}{\partial y}\right)$ with $\zeta$ being the surface height above the mean $(x y)$ plane. It has been assumed that the $x z$ plane is the incident plane and $R(0)$ the Fresnel reflection coefficient of the surface at normal incidence.

The Fresnel reflection coefficient of a surface with dielectric constant $\varepsilon$ is computed by the expression (Jackson 1962):

$$
R(0)=\frac{\varepsilon^{1 / 2}-1}{\varepsilon^{1 / 2}+1}
$$

$\varepsilon$ for sea water is a complex number and is computed as described by Saxton and Lane (1952).

Equation (1) is based on the specular point model (Barrick 1968), which involves derivation of the average number of specular points for a two-dimensionally rough surface and the Gaussian curvature at these points. It is reasonable to interpret (1) as the scattered signal being merely proportional to the probability of the surface having regions tilted at slope- $\tan \theta$, which make them normal to the incident ray. For an isotropic rough surface of Gaussian statistics, (1) becomes

$$
\sigma^{\circ}=\left(1 / S^{2}\right)|R(0)|^{2} \sec ^{4} \theta \exp \left(-\tan ^{2} \theta / S^{2}\right)
$$

where $S^{2}$ is the total variance of slopes. Estimation of surface slope (Phillips 1966) and its substitution in (3) results in a reasonably good agreement with the observed wind dependence of the microwave sea scatter in the quasi-specular range (Barrick 1974). Figure 1 shows the typical wind dependence curves of the backscatter in the quasi specular range for $9 \mathrm{GHz}$ frequency band. The backscattered power predicted by the specular point model is perfectly reflected; hence the backscatter cross-section from these facets is polarization insensitive. The frequency dependence of $\sigma^{\circ}$ is not explicit. The terms $R(0)$ and $S$ enter (3) such that $\sigma^{\circ}$ turns out to be very weakly dependent on frequency. In contrast, a sharp angular dependence is seen. The curve corresponding to angle of incidence of $10^{\circ}$ in figure 1 , seems to separate the curves of different nature of wind dependence. Backscatter decreases with increasing wind speed 
for angles below $10^{\circ}$, whereas it increases for angles above it. For incidence angle of $10^{\circ}$, backscatter cross-section seems to be insensitive to variations in wind speed, which makes it ideal for normalisation purposes.

\section{Result}

Experimental measurements (Jones and Schroeder 1978; Moore and Fung 1979) of scattering coefficients at different frequencies, polarization and angles of incidence have exhibited that a power law relationship of the type $\sigma^{\circ}=a W^{b}$ exists. This is corroborated by the results obtained through the specular point model (figure 1), which shows that $\sigma^{\circ}-W$ relation when plotted in logarithmic scale conform to straight lines. As only the angular dependence is of any consequence, the power law relation in the quasi specular region can be written in generalised form

$$
\sigma^{\circ}=a(\theta) W^{b(\theta)} \text {. }
$$

Such a relation is useful in interpreting data obtained by missions with scatterometer antenna having different depression angles or for the same mission with the multiangle scanning capability.

The functions $a(\theta)$ and $b(\theta)$ were generated by the method of numerical curve fitting applied on values of intercepts and slopes of $\sigma^{\circ} W$ lines (figure 1 ), calculated for incidence angles with an interval of half a degree. Three degree polynomials generated by the points obtained for different incident angles (figures 2 and 3) give excellent fits with RMS errors of only $0.3 \times 10^{-2}$ and $0.2 \times 10^{-1}$ respectively. The functions thus obtained are

$$
a(\theta)=\exp \left(\sum_{i=0}^{3} a_{i} \theta^{i}\right)
$$

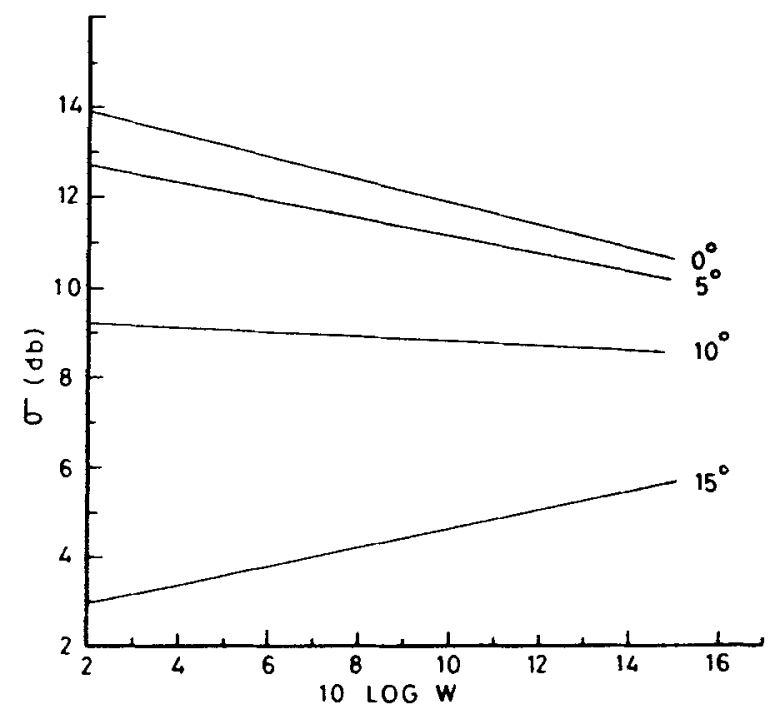

Figure 1. $\sigma^{\circ}$ as a function of $W$ for angles of incidence $0,5,10$ and $15^{\circ}$. 


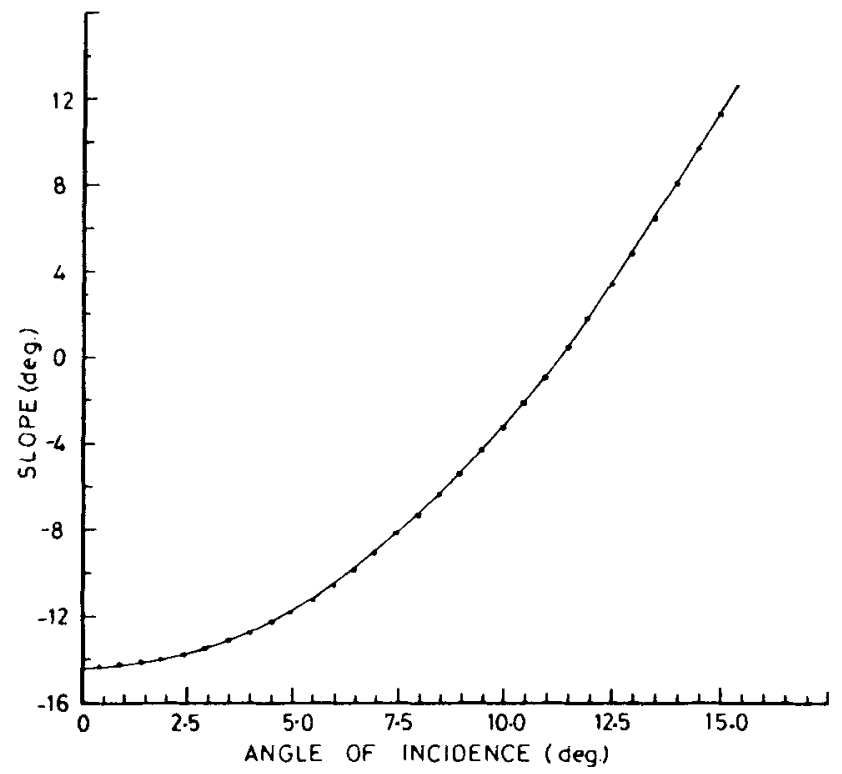

Figure 2. Slopes of the lines of figure $1 \mathrm{vs}$ angies of incidence.

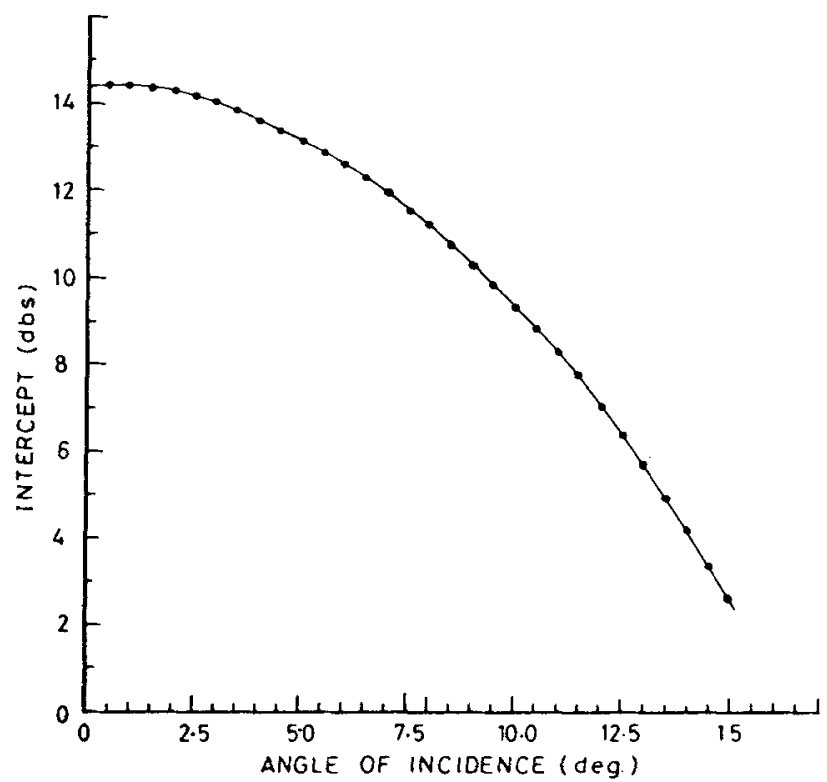

Figure 3. Intercepts of the lines of figure $1 \mathrm{vs}$ angles of incidence.

and $\quad b(\theta)=\tan \left(\sum_{i=0}^{3} b_{i} \theta^{i}\right)$

where, $\theta$ is in degrees and

$$
a_{0}=3.3158 ; a_{1}=-0.30853 \times 10^{-2}
$$




$$
\begin{aligned}
& a_{2}=-0.107584 \times 10^{-1} ; a_{3}=-0.81324 \times 10^{-4} \\
& b_{0}=-14.2883 ; b_{1}=-0.261545 \times 10^{-1} \\
& b_{2}=0.107429 ; b_{3}=0.675045 \times 10^{-3}
\end{aligned}
$$

These coefficients are for $9 \mathrm{GHz}$ frequency.

Similar relations, generated for $14 \mathrm{GHz}$ give $\sigma^{\circ}$ values, which match favourably with the experimental results reported by Brown (1979).

\section{Discussion}

The computation of $\sigma^{\circ}$ required calculation of total variance of surface slopes and Fresnel reflection coefficients for sea water. The sea surface slopes are assumed to be Gaussian and isotropic in their distribution. The crosswind-to-upwind/downwind mean square slope ratio, determined by experimental measurements carried out by Cox and Munk (1954) is as high as 0.75 and hence the assumption that roughness statistics is isotropic is justified, at least as a first approximation. The mean-square slopes of waves longer than the wavelength of the electromagnetic radiation influence the scatter. Hence the returns near the vertical are dominated by large-scale waves, which are not as sensitive to the local wind as the capillary waves. Mid-angle range (angles $>15-20^{\circ}$ ) are better suited for sensing sea surface winds. For such angles, scattering coefficient besides being dependent on angle is also strongly frequency dependent. For these angles, polarization too has to be taken into account. Efforts to develop a similar relationship in the mid angular range are in progress.

\section{Acknowledgements}

The authors are grateful to $\operatorname{Dr} \mathrm{T} A$ Hariharan for constant encouragement and guidance and acknowledge the benefit of their discussions with $\mathrm{Mr} \mathrm{N} S$ Pillai, Microwave components and Sub-systems Division, Dr P S Desai, Dr P C Pandey and other colleagues of the Meteorology Division, Space Applications Centre.

\section{References}

Barrick D E 1968 IEEE Trans. Antennas Propag. AP-16 449

Barrick D E 1974 IEEE Trans. Antennas Propag. AP-22 135

Barrick D E and Peake W H 1968 Radio Sci. 3865

Bass F G, Fuks I M, Kalmykov A I, Ostrovsky I E and Rosenberg A D 1968 IEEE Trans. Antennas Propag. AP-16 554

Brown G S 1979 J. Geophys. Res. 843974

Cox C and Munk W 1954 J. Opt. Soc. Am. 44838

Fung A K 1981 Coherent and incoherent radar scattering from rough surfaces and vegetated areas (ESA SP-166) p. 71

Jackson J D 1962 Classical electrodynamics (New York: John Wiley)

Jones W L and Schroeder L C 1978 Boundary Layer Meteorol. 131333

Jones W L, Schroeder L C, Boggs D H, Bracalente E M, Brown R A, Dome G J, Pierson W J and Wentz F J 1982 J. Geophys. Res. 873297 
Kitaigorodskii S A 1981 Spaceborne synthetic aperture radar for oceanography (Baltimore: Johns Hopkins Univ. Press) 32

Moore R K and Fung A K 1979 Proc. IEEE 671504

Phillips O M 1966 The dynamics of the upper ocean (London: Cambridge University Press)

Saxton J A and Lane J A 1952 Wireless Eng. 29269 